\title{
SEQUELE DI COVID 19 NEL GUSTO E NELL'OLFATTO: UNA BREVE RASSEGNA DELLA LETTERATURA
}

\section{ARTICOLO DI LETTERATURA}

CHAVES, leda Bezerra ${ }^{1}$, FECURY, Amanda Alves², OLIVEIRA, Euzébio de ${ }^{3}$, DENDASCK, Carla Viana ${ }^{4}$, DIAS, Claudio Alberto Gellis de Mattos ${ }^{5}$

CHAVES, leda Bezerra. Et al. Sequele di COVID 19 nel gusto e nell'olfatto: una breve rassegna della letteratura. Revista Científica Multidisciplinar Núcleo do Conhecimento. Anno. 06, Ed. 11, Vol. 01, pp. 150-166. Novembre 2021. ISSN: 24480959, Link di accesso: https://www.nucleodoconhecimento.com.br/salute/sequele-dicovid-19, DOI: 10.32749/nucleodoconhecimento.com.br/salute/sequele-di-covid-19

\section{RIEPILOGO}

I virus sono un essere vivente che non è formato dalla struttura cellulare. I virus possono essere trasmessi per contatto diretto con individui infetti o indirettamente da superfici contaminate. Definito come una nuova varietà di coronavirus, COVID 19 ha fatto dichiarare all'Organizzazione mondiale della sanità (OMS) all'inizio del 2020 una crisi della salute pubblica globale. Considerando l'elevata trasmissibilità di questo virus, è stato necessario adottare misure per prevenire la trasmissione e l'infezione. I sintomi causati da COVID-19 sono di solito febbre, tosse secca, mancanza di respiro (dispnea), stanchezza (affaticamento), mal di gola, mal di testa e diarrea. Altri sintomi, in misura minore sono anosmia (perdita dell'olfatto), iposmia (diminuzione

\footnotetext{
${ }^{1}$ Studente di Scuola Superiore Tecnica Integrata in Alimentazione, Istituto di Istruzione di Base, Tecnica e Tecnologica di Amapá (IFAP).

${ }^{2}$ Biomedicale, Dottorato di Ricerca in Malattie Topiche, Professore e ricercatore del Corso di Medicina del Campus Macapá, Università Federale di Amapá (UNIFAP).

${ }^{3}$ Biologo, Dottore di Ricerca in Malattie Topiche, Professore e ricercatore del Corso di Educazione Fisica, Università Federale di Pará (UFPA).

${ }^{4}$ Teologo, Dottore di Ricerca in Psicoanalisi Clinica. Lavora da 15 anni con metodologia scientifica (metodo di ricerca) nella guida alla produzione scientifica di studenti di master e dottorato. Specialista in ricerche di mercato e ricerche incentrate sulla salute. Dottorando in Comunicazione e Semiotica (PUC SP).

${ }^{5}$ Biologo, Dottore di Ricerca in Teoria e Comportamento, Professore e ricercatore del Corso di Laurea in Chimica dell'Istituto di Educazione Di Base, Tecnica e Tecnologica di Amapá (IFAP) e del Corso di Laurea in Formazione Professionale e Tecnologica (PROFEPT IFAP).
}

RC: 101285

Disponibile in: https://www.nucleodoconhecimento.com.br/salute/sequele-di-covid-19 
dell'odore) e ageusia (perdita del senso del gusto). L'obiettivo di questo era quello di rivedere gli ultimi due anni sulle sequele di COVID 19 nel gusto e nell'olfatto. Una breve revisione della letteratura è stata condotta in articoli scientifici sulle sequele di COVID 19 nel gusto e nell'olfatto, tra il 2020 e il 2021, in portoghese, nel database di ricerca di Google Scholar. Le donne e i giovani sono i più colpiti dalle sequele del gusto e dell'olfatto causate da COVID-19. non esistono trattamenti specifici e scientificamente provati alle varie disfunzioni, ma il più indicato è la pratica dell'allenamento olfattivo. Gli studi relativi alle disfunzioni sono limitati, principalmente, in Brasile e quando vengono analizzati come sequele. La continuità della ricerca scientifica è indispensabile, perché solo questo processo sarà in grado di chiarire i dubbi che ancora esistono e generare trattamenti appropriati per le persone che hanno sviluppato COVID-19.

Parole chiave: Virus, COVID 19, Disosmia, Disgeusia.

\section{INTRODUZIONE}

I virus sono un essere vivente che, a differenza degli altri, non è formato da cellule. Di solito ha un acido nucleico (che può essere DNA o RNA), coperto da una "capsula" proteica. Alcuni sono ancora coperti da "pezzi" di membrana cellulare, che creano un "involucro" intorno ad esso. Sono spesso più piccoli di una cellula e li parassitano, entrando in essi e usando le loro strutture per produrre più virus (sono parassiti intracellulari obbligatori) (Brandão, 2015; They, 2020).

I virus possono essere trasmessi per contatto diretto con individui infetti o indirettamente da superfici contaminate. Vengono trasmessi da un individuo all'altro attraverso goccioline d'acqua sotto forma di vapore rilasciato nel respiro o durante il processo di tosse o starnuto. Le goccioline più piccole rilasciate insieme al vapore acqueo durante questi processi sono sospese nell'aria diffondendo il virus su aree più grandi e più lunghe. (Brasil, 2021). A contatto con uno di questi mezzi di trasmissione i virus invadono le cellule mucose delle vie aeree e delle digestórie (Stephens et al., 2009; Silva et al., 2020). 
Per essere riprodotti i virus si legano alla superficie della membrana cellulare. Dopo questo legame può penetrare nella cellula o può "iniettare" il suo materiale genetico (DNA o RNA) nel suo citoplasma. Una volta allinterno delle cellule il materiale genetico del virus viene copiato più volte, utilizzando le reazioni chimiche che può fare. Allo stesso modo, altre reazioni chimiche della cellula vengono utilizzate per fare le "coperture" delle proteine (capsidi). Così nuovi virus sono montati all'interno di una cellula e, per uscire e invadere nuove cellule, rompere la membrana di coloro che li hanno fabbricati (They, 2020; Silva et al., 2020a).

Definito come una nuova varietà di coronavirus, COVID 19 ha fatto sì che I'Organizzazione Mondiale della Sanità (OMS) dichiarasse all'inizio del 2020 una crisi della salute pubblica globale (Souza et al., 2020). Questi virus, con un alto tasso di trasmissione, sono costituiti da involucri di lipidi e proteine (pezzo di membrana di una cellula) e capsidi proteici, con un singolo nastro di rna (acido nucleico) e con forma a corona esterna (Silva et al., 2020). Fanno parte della famiglia di virus che generano raffreddori comuni a infezioni più gravi, come la Sindrome Respiratoria del Medio Oriente (MERS) e la Sindrome Respiratoria Acuta Grave (SARS) (Brasil, $2021 ; 2021 a)$.

Considerando l'elevata trasmissibilità di questo virus, è necessario adottare misure per prevenire la trasmissione e l'infezione. Tra alcune di queste azioni preventive, evidenziamo la non permanenza in luoghi chiusi e l'isolamento delle persone infette (Stephens et al., 2009).

I modi considerati i più efficienti e per evitare la contaminazione (profilassi) includono: lavaggio delle mani, distanziamento sociale, aerazione degli ambienti e loro esposizione alla luce solare, pulizia di oggetti e superfici, divieto di agglomerati e uso di maschere (bloccanti efficienti di goccioline respiratorie sparse, anche a distanze ridotte con contatto diretto o indiretto) (Garcia, 2020). Queste misure di controllo si sono dimostrate efficaci, attraverso analisi e studi scientifici, nel ridurre la contaminazione e la diffusione virale (Soares et al., 2021). 
I sintomi causati da COVID-19 in generale sono febbre, tosse secca, mancanza di respiro (dispnea), stanchezza (affaticamento), mal di gola, mal di testa e diarrea (Silva et al., 2020). Altri sintomi, in misura minore sono anosmia (perdita dell'olfatto), iposmia (diminuzione dell'olfatto) e ageusia (perdita del senso del gusto) (Iser et al., 2020).

Le sequele possono essere definite come un cambiamento nel funzionamento delle cellule e/o nella forma e nel funzionamento degli organi che causano un difetto permanente o meno nell'individuo (Filho, 2011; Vana e Schiozer, 2013).

Tra le varie sequele riscontrate in individui che hanno avuto COVID19 ci sono quelle di natura psicologica e biologica, come cambiamenti nei sistemi respiratorio, cardiovascolare, possibili cambiamenti cutanei e neurologici (depressione e ansia) (Estrela et al., 2021).

Una serie di ricerche scientifiche indica danni a lungo termine (dopo 6 settimane o persistenti oltre le 6 settimane dall'insorgenza dei sintomi del coronavirus) nel sistema sensoriale, con particolare attenzione alle sequele olfattive e gustative, ad esempio disosmia (percezione distorta degli odori) e disgeusia (percezione distorta dei sapori degli alimenti). Ci possono anche essere disturbi del metabolismo del glucosio (reazioni chimiche), iperlipidemia (aumento della quantità di grasso nel sangue), disregolazione del metabolismo lipidico (reazioni chimiche), disturbo da stress post-traumatico (uno dei tipi di disturbi d'ansia) e capacità polmonare colpita, danni al fegato (fegato) e al sistema renale (reni), e possibilmente nel sistema riproduttivo (Sesapr, 2020; Grendene et al., 2021).

Le disfunzioni olfattive hanno una classificazione quantitativa, in cui vi è anosmia (nessun rilevamento degli odori), iposmia (limitazione nel rilevamento degli odori), iperosmia (aumento della capacità di rilevare gli odori) e disosmia (percezione distorta degli odori) (Cardoso, 2018). Mentre le disfunzioni quantitative del gusto possono essere classificate come ipogeusia (rilevazione parziale del gusto), ipergeusia (aumento della capacità di percezione gustativa), ageusia (perdita totale 
del gusto) e disgeusia (distorsione del gusto) (Barros et al., 2015; Gomes et al., 2020; Machado e Machado, 2020).

La sottoclassificazione qualitativa della disosmia (alterazione olfattiva) è diversa, tra gli esempi si possono trovare parosmia (percezione distorta e sgradevole degli odori), fantosmia (percezione dell'odore in assenza di particelle odorifere) e agnosmia (incapacità di classificare odori diversi) (Cardoso, 2018). La disgeusia (alterazione gustativa) ha una sottoclassificazione qualitativa, anche diversificata, tra gli esempi, parageusia (percezione inadeguata del gusto a causa dello stimolo) e fantogeusia (percezione gustativa sgradevole in assenza di stimolo) (Machado e Machado, 2020).

Nonostante le note, sono necessari ulteriori studi in relazione alle numerose e diverse sequele dovute al covid-19 (Grendene et al., 2021), compresi quelli affetti da sintomi ridotti e asintomatici (Uzun, 2020).

\section{OBIETTIVO}

Fai una revisione della letteratura degli ultimi due anni sulle sequele di COVID 19 nel gusto e nell'olfatto.

\section{METODO}

Una breve revisione della letteratura è stata condotta in articoli scientifici sulle sequele di COVID 19 nel gusto e nell'olfatto, tra il 2020 e il 2021, in portoghese, nel database di ricerca di Google Scholar. Sono state utilizzate le parole chiave (o descrittori) "Gustation e COVID19" e "Smell and COVID 19". Sono stati trovati un totale di 1.777 risultati e 10 articoli sono stati utilizzati per preparare questa breve recensione. Ciò ha permesso di fare la revisione sulla base delle produzioni accademiche più aggiornate (Capes, 2012). 
Articoli con contenuti COVID 19 e sequele nel gusto e nell'olfatto sono stati utilizzati, in portoghese, entro il limite di tempo della recensione. Sono stati esclusi gli articoli che non soddisfacevano questo contenuto e/o periodo di tempo.

\section{RISULTATI E DISCUSSIONE}

La tabella 01 mostra il numero di articoli trovati per descritto e il numero di articoli utilizzati nell'ambito dei criteri metodologici.

Tabella 1 - Numero di articoli trovati nella banca dati in base ai descrittori e agli articoli utilizzati

\begin{tabular}{|c|c|c|c|c|}
\hline BASE DE DADOS & DESCRITOR & \begin{tabular}{|c|} 
NUMERO \\
ARTIGOS \\
ENCONTRADOS \\
\end{tabular} & $\begin{array}{c}\text { NUMERO } \\
\text { ARTIGOS } \\
\text { UTILIZADOS }\end{array}$ & \begin{tabular}{|c|} 
AUTOR E ANO DOS \\
ARTIGOS \\
UTILIZADOS \\
\end{tabular} \\
\hline \multirow{10}{*}{ Google acadêmico } & \multirow{7}{*}{ Gustação e COVID19 } & \multirow{7}{*}{237} & \multirow{7}{*}{7} & PIMENTEL, 2020 \\
\hline & & & & $\begin{array}{l}\text { GRENDENE et al., } \\
2021\end{array}$ \\
\hline & & & & SANTOS et al., 2020 \\
\hline & & & & COSTA et al., 2020 \\
\hline & & & & SOUZA et al., 2021 \\
\hline & & & & $\begin{array}{c}\text { NOGUEIRA et al., } \\
2021\end{array}$ \\
\hline & & & & NUNES et al., 2020 \\
\hline & \multirow{3}{*}{ Olfato e COVID 19} & \multirow{3}{*}{1540} & \multirow{3}{*}{3} & KOSUGI et al., 2020 \\
\hline & & & & BRITO e SLVA, 2020 \\
\hline & & & & $\begin{array}{c}\text { LOPES e DE ABREU, } \\
2021\end{array}$ \\
\hline
\end{tabular}

RC: 101285

Disponibile in: https://www.nucleodoconhecimento.com.br/salute/sequele-di-covid-19 
La tabella - 02 mostra i metodi utilizzati in ogni articolo selezionato per questa revisione, i risultati e le conclusioni trovate.

\begin{tabular}{|c|c|c|c|}
\hline $\begin{array}{c}\text { AUTOR E ANO } \\
\text { DOS ARTIGOS } \\
\text { UTILIZADOS }\end{array}$ & $\begin{array}{l}\text { TIPO DE } \\
\text { MÉTODO }\end{array}$ & RESULTADOS & CONCLUSÃO \\
\hline PIMENTEL, 2020 & $\begin{array}{l}\text { Pesquisa } \\
\text { qualitativa } \\
\text { descritiva, } \\
\text { revisão } \\
\text { bibliográfica da } \\
\text { literatura }\end{array}$ & $\begin{array}{l}\text { Estudos dassificaram as disfunções como: } \\
\text { anosmia/hiposmia ou ageusia e } \\
\text { hipogeusia/disgeusia. Pacientes tiveram os sintomas } \\
\text { disfuncionais tanto no olfato quanto na gustação. } \\
\text { Não são considerados danos permanentes. }\end{array}$ & $\begin{array}{l}\text { Identificação hiposmia, anosmia, fantosmia e } \\
\text { parosmia, com preval ência entre } 22,7 \% \text { a } 88,8 \% \text {, } \\
\text { associadas ou não hipogeu sia e ag eusia Futuros } \\
\text { estudos epi demiológicos devem esclarecer como } \\
\text { esses sintomas são causados. }\end{array}$ \\
\hline $\begin{array}{l}\text { SANTOS et al }= \\
2020\end{array}$ & $\begin{array}{c}\text { Revisão } \\
\text { Integrativa da } \\
\text { literatura }\end{array}$ & $\begin{array}{l}\text { Preval ência das disfunções sensoriais provocadas } \\
\text { pela COVID-19. Anosmia e disgeusia como } \\
\text { sintomas neurológicos mais frequentes. Pacientes } \\
\text { que tiveram quadro leve a moderado de COVID-19. } \\
\text { Aumento da idade dos pacientes e quadros mais } \\
\text { severos da doença representam menos al terações. }\end{array}$ & $\begin{array}{l}\text { Sintese de informações de vários paises, } \\
\text { caracterizando as disfunções olfativas e gustativas } \\
\text { como sintomas iniciais. Repercu ssões após alta dos } \\
\text { pacientes. Necessários estudos sobre as } \\
\text { probl emáticas e investigações mais detalhadas. Os } \\
\text { distúrbios poderão ser estudados em um periodo de } \\
\text { tempo maior. }\end{array}$ \\
\hline $\begin{array}{c}\text { NOGUEIRA et al ., } \\
2021\end{array}$ & $\begin{array}{l}\text { Revisão } \\
\text { descritiva }\end{array}$ & $\begin{array}{l}\text { A infecção nos neurônios causa sintomas } \\
\text { neurológicos como anosmia. Não há explicação } \\
\text { exata acerca da ocorrência dos distúrbios olfatórios. } \\
\text { Danos no sistema nervoso central e epitélio nasal, } \\
\text { cau sam a di sosmia e a disgeusia. }\end{array}$ & $\begin{array}{l}\text { Os problemas olfativos e gustativo ocorrem } \\
\text { previamente em diferentes intensi dades. Não há } \\
\text { evidências cientificas para o tratamento desses } \\
\text { distúrbios. É importante a realização de estudos } \\
\text { acerca do assunto a fim de que se melhore os } \\
\text { procedimentos no tratamento nos casos, } \\
\text { principalmente, nos casos com sequelas de longo } \\
\text { prazo. }\end{array}$ \\
\hline $\begin{array}{c}\text { COSTA et al., } \\
2020\end{array}$ & $\begin{array}{l}\text { Revisão } \\
\text { Sistemática }\end{array}$ & $\begin{array}{l}\text { Sintomas neurológicos que ocorrem no sistema } \\
\text { nervoso periférico causam as disfunções olfativa e } \\
\text { gustativa. Pacientes com quadro leve a moderado } \\
\text { apresentaram hipogeusia e hiposmia, não há } \\
\text { registros do inicio, duração e resolução dos } \\
\text { sintomas. Os pacientes com perda de olfato tiveram } \\
\text { menor taxa de internação, comparados com os } \\
\text { pacientes sem perda de olfato. }\end{array}$ & $\begin{array}{l}\text { Os distúrbios olfativos e/ou gustativos podem } \\
\text { ocorrer em intensidades variáveis, com inicio junto } \\
\text { com os sintomas prévios da infecção. Não há } \\
\text { evidências cientificas acerca do tratamento destes } \\
\text { distúrbios. }\end{array}$ \\
\hline
\end{tabular}




\begin{tabular}{|c|c|c|c|}
\hline $\begin{array}{c}\text { BRITO e SIVA, } \\
2020\end{array}$ & $\begin{array}{c}\text { Revisão da } \\
\text { literatura }\end{array}$ & $\begin{array}{l}\text { Associação de manifestações neurológicas e a } \\
\text { COVID-19. Entre elas os distúrbios do olfato e } \\
\text { paladar, como a hiposmia, a anosmia, a disgeusia, } \\
\text { hipogeusia e ageusia. Os mecanismos exatos da } \\
\text { neuroinvasão causada pela COVID-19 ainda são } \\
\text { desconhecidos. }\end{array}$ & $\begin{array}{l}\text { Ocorre uma inci dência maior de danos no si stema } \\
\text { nervoso em quadros agudos da virose. Entretanto a } \\
\text { classificação dos casos agudos e crônicos ainda hoje } \\
\text { não é consenso. São necessários mais estu dos sobre } \\
\text { as sequelas a longo prazo. }\end{array}$ \\
\hline $\begin{array}{c}\text { SOUZA et al., } \\
2021\end{array}$ & Relato de casos & $\begin{array}{c}\text { Ano smia nos pacientes é uma manifestação } \\
\text { sintomal ógica recorrente. Fundamental a descrição } \\
\text { de relatos de casos a fim de esclarecer as } \\
\text { ocorrências de sequel as gustativas olfativas com } \\
\text { objetivo de realializar o manejo da recuperação. }\end{array}$ & $\begin{array}{l}\text { As disfunções devem ser estudadas com maior rigor } \\
\text { e por maior periodo de tempo. A COVID-19 afeta } \\
\text { as células do epitélio olf ativo, possivelmente } \\
\text { alterando a neurotransmissão. }\end{array}$ \\
\hline $\begin{array}{l}\text { LOPES e DE } \\
\text { ABREU } 2021\end{array}$ & $\begin{array}{c}\text { Metodologia para } \\
\text { tratamento }\end{array}$ & $\begin{array}{c}\text { Possiveis danos neurológicos em nivel celular } \\
\text { decorrentes da COVID-19. Pacientes recuperados } \\
\text { ainda relatam alterações, que podem ser } \\
\text { irreversiveis, nos sentidos olf ativo e gustativo. }\end{array}$ & $\begin{array}{l}\text { As sequelas causadas pela doença podem ser de } \\
\text { cunho neurológicos, em nivel celular ou não. Isso } \\
\text { pode causar transtornos, sindromes e outras } \\
\text { variáveis futuramente. }\end{array}$ \\
\hline $\begin{array}{c}\text { NUNES et al., } \\
2020\end{array}$ & $\begin{array}{l}\text { Revisão } \\
\text { Integrativa }\end{array}$ & $\begin{array}{c}\text { Disfunções olfativas com preval ência entre } 30 \text { a } \\
88 \% \text { dos pacientes com COVD-19 englobados nos } \\
\text { diferentes estudos. Relados por pacientes com } \\
\text { quadros leves e moderados da doença. Os } \\
\text { transtornos como anosmia, hiposmiahipogeusia, e } \\
\text { disgeusia são sintomas frequentes nos pacientes } \\
\text { com COVID-19. }\end{array}$ & $\begin{array}{l}\text { O envolvimento do virus com o sistema nervoso é, } \\
\text { até então, inconclusivo. Entender os sintomas } \\
\text { neurológicos, principalmente em quadros mais } \\
\text { graves, auxilia nas intervenções e nos prognósticos } \\
\text { médicos. }\end{array}$ \\
\hline $\begin{array}{c}\text { GRENDENE et } \\
\text { al., } 2021\end{array}$ & $\begin{array}{c}\text { Revisão } \\
\text { bibliográfica }\end{array}$ & $\begin{array}{c}\text { Aponta que existem poucos estudos revisados sobre } \\
\text { a ocorrência ou prevalência das sequelas de longo } \\
\text { prazo da COVID-19. Há algumas evidências de que } \\
\text { disfunções como a disosmia e disgeusia são } \\
\text { sequel as de longo prazo associadas à COVID-19. A } \\
\text { mai oria não trabalha com periodos de } \\
\text { acompanhamento indi cativos de sequelas de longo } \\
\text { prazo. }\end{array}$ & $\begin{array}{l}\text { Não se sabe muito sobre estratégias para tratamento } \\
\text { para este virus. Pesquisas clíni cas estão sendo } \\
\text { realizadas para descobrir novos tratamentos. } \\
\text { Prevenção ainda é a melhor forma de lidar. }\end{array}$ \\
\hline $\begin{array}{c}\text { KOSUGI et al., } \\
2020\end{array}$ & $\begin{array}{l}\text { Quali quantitativa } \\
\text { por questionário }\end{array}$ & $\begin{array}{c}\text { Induidos } 253 \text { pacientes, de todas as regiões } \\
\text { brasileiras, uma maioria apresentou anosmia e } 13 \text {, } \\
2 \% \text { não tiveram recuperação da per da olfativa. } \\
\text { Alteração no paladar não está incluida na pesquisa. }\end{array}$ & $\begin{array}{l}\text { Anosmia apresenta menor taxa de recu peração e } \\
\text { duração mai or em pacientes por COVID-19 } \\
\text { positivos do que nos negativos. Hiposmia teve } \\
\text { maior taxa de recuperação do que a anosmia. }\end{array}$ \\
\hline
\end{tabular}

Attraverso studi Pimentel (2020) basati su 15 pubblicazioni che hanno descritto rapporti clinici in Europa all'inizio del 2020, sono stati identificati casi di iposmia, anosmia, fantosmia e parosmia, con prevalenza tra il $22,7 \%$ e l' $88,8 \%$, associati o meno a ipogeusia ed ageusia.

L'ampiezza dei tassi di sintomi sensoriali / sequele è dovuta alla differenza tra gli studi, così come gli strumenti utilizzati da queste varie ricerche scientifiche e persino le differenze genetiche nella popolazione (Pimentel, 2020). Le differenze di popolazione mostrano che i tassi di insorgenza di anomalie olfattive del gusto sono più elevati, ad esempio, nelle popolazioni in Europa, Nord Africa, Asia meridionale e Asia occidentale, rispetto ai tassi più bassi presentati dai pazienti in Asia orientale (Santos et al., 2020). Le donne e i giovani sembrano essere i più colpiti da disturbi 
olfattivi e gustativi (Costa et al., 2020; Nascimento, 2020). Questa differenza si verifica probabilmente perché c'è una differenza nella reazione fisiologica di difesa tra gruppi di età e generi della specie umana (Cardoso et al., 2020).

Lo studio di Santos et al. (2020) evidenzia che le disfunzioni olfattive e gustative sono sintomi iniziali e che hanno maggiori ripercussioni dopo la dimissione dei pazienti.

II trattamento ospedaliero dei pazienti termina prima della comparsa delle sequele, probabilmente perché c'è una carenza storica di posti vacanti e professionisti della salute in Brasile. L'aumento del flusso ospedaliero di pazienti durante la pandemia sembra aver aggravato la situazione, impedendo un follow-up specifico e approfondito, in quanto le cure erano già precarie (Santo et al., 2021).

Nella revisione integrativa di Nogueira et al. (2021) è stato identificato che i problemi olfattivi e gustativi si verificano in precedenza a intensità diverse. Non ci sono prove scientifiche per il trattamento di questi disturbi.

Costa et al. (2020) hanno identificato che i disturbi olfattivi e / o gustativi possono verificarsi a intensità variabili, con insorgenza insieme a precedenti sintomi di infezione. Non ci sono prove scientifiche sul trattamento di questi disturbi.

II sistema immunitario umano varia in base ad alcune caratteristiche fisiche e fisiologiche (Cardoso et al., 2020). L'intensità dei sintomi può verificarsi a causa di queste variazioni. Nelle donne gli ormoni prodotti nei cicli mestruali, quelli presenti nella pillola contraccettiva e nel trattamento della sostituzione ormonale (per combattere i sintomi della menopausa) influenzano il sistema di difesa, rendendolo diverso da quello degli uomini (Rogero et al., 2021).

L'età è anche un fattore per la differenza nell'insorgenza dei sintomi. In contrapoint a Costa et al. (2020) e Nascimento (2020), un'età più avanzata sembra essere più suscettibile ai sintomi causati dal virus. Un intestino più vecchio ha in genere un minore assorbimento di sostanze nutritive e produzione di cellule di difesa. La 
differenza di concetto tra gli autori può essere correlata al tipo specifico di sintomo (Rogero et al., 2021).

Lo stress influisce anche sulla funzione di difesa immunitaria. II sistema nervoso influenza la produzione e il rilascio di ormoni che possono mediare questa funzione (Rogero et al., 2021). Le persone più giovani, a causa della loro esperienza più breve, possono essere più colpite dallo stress.

Per quanto riguarda i trattamenti studiati nell'azione contro i disturbi olfattivi e gustativi, la letteratura presenta l'uso di corticosteroidi (azione antinfiammatoria) dell'apparato orale e orale, allenamento olfattivo, vitamina $\mathrm{A}$ e citrato di sodio. $\mathrm{Ci}$ sono altre alternative terapeutiche, ma quelle presentate sopra sono più favorevoli all'efficacia. Tuttavia, ci sono prove insufficienti o sufficienti circa l'efficacia di questi trattamenti (Cardoso et al., 2020; Costa et al., 2020; Felipe et al., 2021; Neta et al., 2021).

Attraverso una revisione della letteratura (Brito e Silva, 2020), ritengono che vi sia una maggiore incidenza di danni al sistema nervoso nei casi acuti del virus. Tuttavia, la classificazione dei casi acuti e cronici non è ancora consensuale.

Souza et al. (2021) hanno concluso che le disfunzioni dovrebbero essere studiate in modo più rigoroso e per un periodo di tempo più lungo. COVID-19 colpisce le cellule dell'epitelio olfattivo, alterando eventualmente la neurotrasmissione.

Lo studio di Lopes e De Abreu (2021) riporta che le sequele causate dalla malattia possono essere di natura neurologica, a livello cellulare o meno. Ciò può causare disturbi, sindromi e altre variabili in futuro.

Le disfunzioni olfattive e gustative, in generale, si verificano in modo associato, poiché i sensi olfattivo e gustativo (sensi chimici, percepiscono le sostanze nel cavo nasale e orale) lavorano insieme e le sensazioni gustative dipendono esclusivamente dall'olfatto. Le sensazioni sensoriali si verificano a causa dellinterazione delle molecole con i recettori dell'olfatto e del gusto. I recettori del gusto (che si trovano 
nelle regioni della lingua, del palato, della faringe, dell'epiglottide e dell'esofago) sono responsabili dell'invio di informazioni gustative attraverso impulsi nervosi (comunicazione tra cellule, neuroni) al sistema nervoso centrale. Nell'olfatto, le informazioni olfattive vengono trasmesse anche al sistema nervoso centrale attraverso i neuroni che comunicano tramite correnti elettriche (sinapsi) (Neto et al., 2011; Oliveira, 2014).

II virus COVID19 provoca lesioni alle cellule neurali olfattive, perché nell'epitelio nasale ci sono un'elevata quantità di enzimi che facilitano la diffusione e la replicazione di esso. In risposta all'infezione il sistema immunitario "attiva" il sistema di difesa dalle citosine (proteine), il cosiddetto effetto citopatico, che può compromettere il processo di neurotrasmissione, causando la perdita olfattiva. Ciò si verifica anche con il gusto, dove il processo infiammatorio (effetto citopatico) diminuisce la vita delle cellule recettrici delle papille gustative (Cardoso et al., 2020; Felipe et al., 2021).

Le analisi scientifiche dimostrano la probabilità che il virus raggiunga direttamente l'epitelio nasale, non i neuroni. Le cellule dell'epitelio nasale mantengono il cosiddetto equilibrio ionico del muco, da cui dipendono i neuroni per fare le sinapsi fino al sistema nervoso centrale. Quando questo equilibrio è compromesso l'odore cessa di funzionare come dovrebbe, possono verificarsi danni ai neuroni olfattivi. Tuttavia, non vi è alcuna prova se l'anosmia derivi direttamente dal virus o se sia il risultato di una risposta immunologica (Nishioka, 2020).

In senso gustativo, le cellule recettrici gustative non sono influenzate dal virus, ma piuttosto le cellule di supporto, il che può spiegare i casi di perdita del gusto. L'epitelio olfattivo ha la capacità di rigenerarsi, cioè l'anosmia è considerata reversibile, tuttavia, i pazienti con recupero lento da questa disfunzione possono presentare un altro disturbo, la parosmia, in cui i sapori (dolce, amaro, salato, acido) vengono recuperati, ma alcuni odori e aromi diventano sgradevoli (Nishioka, 2020).

Nunes et al. (2020), nella loro recensione, hanno scoperto che il coinvolgimento del virus con il sistema nervoso è, fino ad allora, inconcludente. Comprendere i sintomi 
neurologici, specialmente nelle condizioni più gravi, aiuta negli interventi e nelle prognosi mediche.

II coinvolgimento del virus con il sistema nervoso sembra essere inconcludente. Attualmente, ci sono due teorie principali che spiegano gli impatti neurologici di COVID-19 sugli esseri umani, la prima corrisponde all'azione del virus nella disregolazione immunitaria, come la sovrapproduzione di citosine e quindi causare sintomi neurologici. Indicare la possibilità che il virus si diffonda attraverso il sangue e le vie neurali al sistema nervoso. II secondo suggerisce che l'infezione nel sistema nervoso centrale avviene attraverso neuroni periferici infettati dal virus (Felipe et al., 2021).

Brito e Silva (2020), Pimentel (2020), Santos et al. (2020) e Nogueira et al. (2021) concordano sulla poca conoscenza che esiste oggi e propongono la continuità degli studi sui sintomi del COVID 19. Questo è importante perché attraverso studi scientifici sarà possibile definire le cause dei meccanismi che causano cambiamenti olfattivi-gustativi e fornire una base per la ricerca di trattamenti appropriati per le persone colpite (Nishioka, 2020; Santos et al., 2020; Felipe et al., 2021; Lima et al., 2021; Oliveira et al., 2021).

Nella revisione della letteratura di Grendene et al. (2021) la conclusione indica che non si sa molto sulle strategie per il trattamento di questo virus. Ecco perché viene condotta una ricerca clinica per scoprire nuovi trattamenti. La prevenzione oggi è ancora il modo migliore per affrontare l'infezione.

Le misure di prevenzione contro il virus includono: l'igiene delle mani spesso, fatta lavando con acqua e sapone (rompendo la parete lipidica del virus e la uccide) e / o usando alcol etilico al 70\%, evitando le mani con occhi, naso e bocca, praticando l'etichettatura respiratoria (cura quando si tossisce o starnutisce), indossando maschere per il viso (barriera fisica contro le goccioline appese), distanziamento sociale di almeno un metro, disinfezione di oggetti e superfici, tenere sintomatici in isolamento, estetizzarsi al chiuso, evitare strette di mano e abbracci ed evitare il 
contatto sociale. Tutti sono indicati secondo le basi scientifiche (Stephens et al., 2009; Baptista e Fernandes, 2020; Garcia, 2020; Soares et al., 2021; Spdm, 2021)

In relazione all'isolamento sociale, gli studi indicano la sua efficacia e l'aiuto nel ridurre i tassi di infezione, tuttavia, dovrebbe essere fatto in associazione con altre meditazioni di contenimento del virus (Who, 2021). Ci sono anche prove sull'aerazione di luoghi chiusi quando esposti alla luce solare e tenerli ventilati, perché tali ambienti contengono le goccioline di vapore acqueo da tosse, starnuti e l'atto stesso di parlare di individui, (Opas, 2021). L'uso delle mascherine è scientificamente provato, è noto che questo DPI (dispositivi di protezione individuale) è una barriera fisica, un muro, contro il virus COVID-19 (Salles et al, 2021). Inoltre, si sottolinea che anche dopo la vaccinazione le persone non dovrebbero smettere di usare/applicare i meccanismi di prevenzione contro il virus (Brasil, 2021a).

Kosugi et al. (2020) hanno condotto un sondaggio online e hanno concluso che I'anosmia ha un tasso di recupero inferiore e una durata più lunga nei pazienti positivi al COVID-19 rispetto ai pazienti negativi. L'iposmia ha avuto un tasso di recupero più elevato rispetto all'anosmia.

Nell'iposmia, poiché si tratta di una perdita parziale, è possibile "allenare" l'odore. Gli odori e gli oli essenziali vengono utilizzati due volte al giorno per sei mesi per questo scopo. Perdita già più grave, anosmia, se non c'è recupero istantaneo il trattamento è farmacologico e prolungato (Pereira et al., 2020). Questo probabilmente spiega la differenza nei tassi di recupero di entrambi i sequel

\section{CONCLUSIONI}

Le sequele di COVID-19 sono diversi disturbi che compromettono i sensi sensoriali (olfatto e gusto) in diversi gradi, influenzando il benessere e la vita quotidiana di migliaia di persone.

Le donne e i giovani sono i più colpiti da queste disfunzioni. Non è noto esattamente come il virus causi sintomi neurologici nei pazienti e il mezzo esatto con cui si 
verificano disfunzioni. Inoltre, non esistono trattamenti specifici e scientificamente provati alle varie disfunzioni, ma il più indicato è la pratica dell'allenamento olfattivo.

Gli studi relativi alle disfunzioni sono limitati, principalmente, in Brasile e quando vengono analizzati come sequele, cioè studiati dopo un certo periodo dopo il recupero dei pazienti COVID-19. La continuità della ricerca scientifica è indispensabile, perché solo questo processo sarà in grado di chiarire i dubbi che ancora esistono e generare trattamenti appropriati per le persone che hanno sviluppato COVID-19 e sono state lasciate con alcuni dei vari tipi di sequele, comprese quelle sensoriali.

\section{RIFERIMENTI}

BAPTISTA, A. B.; FERNANDES, L. V. COVID-19, análise das estratégias de prevenção, cuidados e complicações sintomáticas. Revista Desafios, v. 7, p. 1-10, 2020.

BARROS, Ó. D. et al. Disgeusia: a propósito de um caso clínico. Revista Portuguesa De Medicina Geral E Familiar, v. 31, n. 4, p. 272-276, 2015.

BRANDÃO, R. E. L. Vírus e Retrovírus: Contributo para a Evolução das Espécies. 2015. 61p. (Mestrado). Faculdade de Ciências da Saúde, Universidade Fernando Pessoa, Porto PT.

BRASIL. Coronavírus - Como é transmitido? , Brasília DF, 2021. Disponível em: < https://www.gov.br/saude/pt-br/coronavirus/como-e-transmitido >. Acesso em: 06 set 2021.

- Doenças ocasionadas por vírus respiratórios emergentes, incluindo o COVID-19. Brasília DF, 2021a. Disponível em: < https://www.unasus.gov.br/cursos/curso/46164 >. Acesso em: 06 set 2021.

BRITO, W. G. F.; SILVA, J. P. D. O. Impactos neuropatológicos do COVID-19. Brazilian Journal of Health Review, v. 3, n. 3, p. 4227-4235, 2020. 
CAPES. Treinamento no uso do Portal de Periódicos. Brasilia DF, 2012. Disponível em: < https://www.fca.unesp.br/Home/Biblioteca/portal-capes.pdf >. Acesso em: 06 set 2021.

CARDOSO, A. R. Olfato como Marcador Biológico. 2018. 21p. (Mestrado). Universidade de Lisboa, Lisboa PT.

CARDOSO, M. D. C. et al. Anosmia e disgeusia no paciente com coronavírus: revisão narrativa. REAS/EJCH, v. 46, p. 1-8, 2020.

COSTA, K. V. T. D. et al. Desordens olfativas e gustativas na COVID-19: uma revisão sistemática. Braz J Otorhinolaryngol., v. 86, n. 6, p. 781-792, 2020.

ESTRELA, M. C. A. et al. Covid-19: sequelas fisiopatológicas e psicológicas nos pacientes e na equipe profissional multidisciplinar. Brazilian Journal of Development, v. 7, n. 6, p. 59138-59152, 2021.

FELIPE, L. P. et al. Compreensão das manifestações neurológicas induzidas por infecções pelo novo coronavírus: uma revisão integrativa. REAID, v. 95, n. 36, p. e21137, 2021.

FILHO, N. D. A. O que é saúde? Rio de Janeiro RJ: Editora Fiocruz, 2011. 160p.

GARCIA, L. P. Uso de máscara facial para limitar a transmissão da COVID-19. Epidemiol. Serv. Saude, v. 29, n. 2, p. 1-4, 2020.

GOMES, D. R. D. P. et al. Avaliação do paladar de idosos e sua relação com estado nutricional e hábitos alimentares. Pan American Journal of Aging Research, v. 8, n. 1, p. 1-8, 2020.

GRENDENE, C. S.; GULO, R. B.; BETIOL, R. S. M. Coronavírus (covid-19): história, conhecimento atual e sequelas de longo prazo. Revista Corpus Hippocraticum, v. 1, n. 1, p. 1-14, 2021. 
ISER, B. P. M. et al. Definição de caso suspeito da COVID-19: uma revisão narrativa dos sinais e sintomas mais frequentes entre os casos confirmados. Epidemiol. Serv. Saude, Brasília, v. 29, n. 3, p. 1-11, 2020.

KOSUGI, E. M. et al. Recuperação incompleta e tardia da perda súbita do olfato na COVID-19. Braz. j. otorhinolaryngol., v. 86, n. 4, p. 490-496, 2020.

LIMA, J. H. C. et al. Covid-19 e os danos ao aparelho olfatório causando anosmia. Revista Científica Multidisciplinar, v. 2, n. 8, p. e28665, 2021.

LOPES, D. O.; DE ABREU, F. Eletroterapia IVL no tratamento de Covid-19 e sequelas no sistema nervoso central. Brazilian Journal of Development, v. 7, n. 4, p. $42332-423402021$.

MACHADO, A. L. M.; MACHADO, M. C. M. Disgeusia: Revisão de Literatura. 2020. 56p. (Graduação). Universidade de Uberaba, Uberaba MG.

NASCIMENTO, M. A. Alteração das funções sensoriais de olfato e paladar e seus correlatos clínicos e funcionais em indivíduos com Covid-19. 2020. 71p. (Mestrado). Universidade Federal do Rio Grande do Norte, Santa Cruz.

NETA, F. I. et al. Pathophysiology and possible treatments for olfactory-gustatory disorders inpatients affected by COVID-19. Current Research in Pharmacology and Drug Discovery, v. 2, p. 1-11, 2021.

NETO, F. X. P. et al. Anormalidades sensoriais: Olfato e paladar. Arquivos Int. Otorrinolaringol., v. 15, n. 3, p. 350-358, 2011.

NISHIOKA, S. D. A. A perda de olfato na COVID-19 já tem explicação - pelos menos em $\quad 2020$ parte. Disponível em: < https://www.unasus.gov.br/especial/covid19/markdown/335 >. Acesso em: 30 out 2021. 
NOGUEIRA, J. F. et al. Distúrbios olfatórios decorrentes de infecção por SARSCoV-2: fisiopatologia, fatores de risco e possíveis intervenções. Research, Society and Development, v. 10, n. 11, p. 1-7, 2021.

NUNES, L. T. D. et al. Principais Manifestações Neurológicas decorrentes do COVID-19: uma revisão integrativa. Revista Saúde Coletiva, v. 10, n. 59, p. 42484254, 2020.

OLIVEIRA, A. A. D. et al. Análise dos principais fatores de risco preexistentes em pacientes diagnosticados com a COVID-19 no Amapá, Amazônia, Brasil. Revista Científica Multidisciplinar Núcleo do Conhecimento, v. 17, p. 56-72, 2021. Disponível em: < https://www.nucleodoconhecimento.com.br/saude/riscopreexistentes $>$.

OLIVEIRA, F. V. Aromas: contextualizando o ensino de Química através do olfato e paladar. 2014. 137 p. (Mestrado). Universidade de Santa Maria, Santa Maria RS.

OMS. Série de documentos de informação sintéticos do escritório regional da oms para a áfrica sobre a COVID-19. Africa, p. 1-6, 2021. Disponível em: < https://apps.who.int/iris/bitstream/handle/10665/338819/WHO-AF-ARD-DAK-102021-por. pdf?sequence=1\&isAllowed=y >. Acesso em: 30 out 2021.

OPAS. Roteiro para melhorar e garantir a boa ventilação de ambientes fechados no contexto da doença causada pelo novo coronavirus, COVID-19. Washington D.C., 2021. Disponível em: < https://iris.paho.org/handle/10665.2/53938 >. Acesso em: 01 out 2021.

PEREIRA, A. C. C. M. et al. Revisão bibliográfica: Anosmia no COVID-19. Revista Cien, v. 15, n. 2, p. 96-, 2020.

PIMENTEL, B. N. As disfunções olfativas e gustativas como apresentação clínica da COVID-19. Research, Society and Development, v. 9, n. 8, p. e64985072, 2020. 
ROGERO, M.; NATACCI, L.; AMANCIO, O. Nutrição \& Imunidade. São Paulo SP: SBAN, 2021. 27p.

SANTO, D. M. N. D. E. et al. Desafios do enfermeiro do Centro Cirúrgico frente à pandemia da COVID-19 e transição de uma sala cirúrgica em unidade de terapia semi-intensiva. REAS, v. 13, n. 6, p. 1-6, 2021.

SANTOS, I. H. A. et al. Disfunções olfativas e gustativas na COVID-19. Research, Society and Development, v. 9, n. 12, p. e42591211363, 2020.

SESAPR. Reabilitação do paciente pós tratamento de infecção por SARS- CoV-2. Curitiba $\quad 2020 . \quad$ PR, Disponível em: https://www.saude.pr.gov.br/sites/default/arquivos_restritos/files/documento/202012/Nota\%20orientativa\%20n\%C2\%BA\%2053\%20reabilita\%C3\%A7\%C3\%A3o.pdf >. Acesso em: 09 set 2021.

SILVA, A. W. C. et al. Caracterização clínica e epidemiologia de 1560 casos de COVID-19 em Macapá/AP, extremo norte do Brasil. Research, Society and Development, v. 9, n. 8, p. 1-21, 2020a. Disponível em: < https://rsdjournal.org/index.php/rsd/article/view/5499/4641 >.

SILVA, A. W. C. et al. Perfil epidemiológico e determinante social do COVID-19 em Macapá, Amapá, Amazônia, Brasil. Revista Científica Multidisciplinar Núcleo do Conhecimento, v. 4, n. 4, p. 05-27, 2020. Disponível em: < https://www.nucleodoconhecimento.com.br/saude/covid-19-em-macapa >.

SOARES, K. H. D. et al. Medidas de prevenção e controle da covid-19: revisão integrativa. Revista Eletrônica Acervo Saúde, v. 13, n. 3, p. 1-11, 2021. Disponível em: < https://doi.org/10.25248/reas.e6071.2021 >.

SOUZA, F. D. S. et al. "Anarquia do paladar" e anosmia pós-COVID-19: Seriam danos permanentes? Relato de casos. Revista de Ciências Biológicas e da Saúde, v. 4, n. 1, p. 1-6, 2021. 
SOUZA, K. O. et al. Covid-19 e o cenário atual da Cidade de Castanhal PA, Brasil. Research, Society And Development, v. 9, n. 8, p. 1-17, 2020. Disponível em: < https://rsdjournal.org/index.php/rsd/article/view/5717/4998 >.

SPDM. HMB explica por que a higienização das mãos auxilia na prevenção da Covid-19. São Paulo SP, 2021. Disponível em: < https://www.spdm.org.br/saude/noticias/item/3390-hmb-explica-por-que-ahigienizacao-das-maos-auxilia-na-prevencao-da-covid-19 >. Acesso em: 04 out 2021.

STEPHENS, P. R. S. et al. Virologia. In: MOLINARO, E. M.;CAPUTO, L. F. G., et al (Ed.). Conceitos e métodos para a formação de profissionais em laboratórios de saúde. Rio de Janeiro RJ: EPSJV, v.4, 2009. p.125 - 220.

THEY, N. H. Você sabe o que é um vírus? Microbiologando Porto Alegre RS, 2020. Disponível em: < https://www.ufrgs.br/microbiologando/voce-sabe-o-que-eum-virus/ >. Acesso em: 06 set 2021.

UZUN, A. C. D. S. B. Sequelas da Covid-19 vão além da fadiga, ressalta especialista. Campo Grande MS, 2020. Disponível em: < http://www.ms.gov.br/sequelas-da-covid-19-vao-alem-da-fadiga-ressalta-especialista/ >. Acesso em: 06 set 2021.

VANA, L. P. M.; SCHIOZER, W. Sequelas de queimaduras: nova classificação. Rev Bras Queimaduras, v. 12, n. 3, p. 192, 2013.

Inviato: Novembre, 2021.

Approvato: Novembre 2021. 